\title{
ARTICLE
}

\section{Acute and long-term effects of electroconvulsive therapy on human dentate gyrus}

\author{
Akihiro Takamiya $^{1,2}$, Eric Plitman ${ }^{3,4}$, Jun Ku Chung ${ }^{5,6}$, Mallar Chakravarty ${ }^{3,4}$, Ariel Graff-Guerrero ${ }^{5,6,7,8}$, Masaru Mimura ${ }^{1}$ and \\ Taishiro Kishimoto ${ }^{1}$
}

Electroconvulsive therapy (ECT) is the most effective treatment for severe depression, although the underlying mechanisms remain unclear. Animal studies have consistently shown that electroconvulsive stimulation induces neuroplastic changes in the dentate gyrus. To date, few studies have investigated the effect of ECT on human hippocampal subfields. In the current study, structural magnetic resonance imaging (MRI) was conducted in 25 patients with major depressive episodes at 3 time points: before ECT (TP1), after 1 week of the last ECT (TP2) and after 3 months of the last ECT (TP3). Twenty healthy controls were scanned twice with an interval similar to patients between TP1 and TP2. Volumetric analyses of the cornu ammonis (CA)4/dentate gyrus (DG) were performed using the MAGeT-Brain (Multiple Automatically Generated Templates) algorithm. Clinically remitted patients after ECT showed larger volume increases in the right CA4/DG than non-remitted patients. Volume increases in the right CA4/DG were negatively associated with age. Increased CA4/DG volumes after ECT returned to baseline levels after 3 months irrespective of clinical state. ECT-induced volume increase in the CA4/DG was associated with age and clinical remission. These findings are consistent with the neurotrophic processes seen in preclinical studies. Neuroplastic change in the CA4/DG might mediate some of the short-term antidepressant effects of ECT.

Neuropsychopharmacology (2019) 44:1805-1811; https://doi.org/10.1038/s41386-019-0312-0

\section{INTRODUCTION}

Electroconvulsive therapy (ECT) is one of the most effective treatments for severe depression [1]. ECT is effective not only for major depressive disorder (MDD) but also for bipolar depression [2]. In addition, ECT induces rapid alleviation of suicidal intent [3]. Considering these clinical important aspects, a better understanding of the underlying mechanisms of ECT may lead to the development of novel pharmacological and neuromodulation treatments for depression.

Preclinical studies have consistently reported that electroconvulsive stimulation (ECS), an animal model of ECT, enhances adult hippocampal neurogenesis in both rodent [4] and non-human primate [5]. ECS also promoted other neuroplastic effects, including gliogenesis, angiogenesis, and synaptogenesis [6], and ECS increased volume in the dentate gyrus (DG) in the hippocampus [7-9]. Moreover, ECS seemed to be required hippocampal neuroplasticity for its antidepressant effect [10].

Our recent meta-analysis revealed that ECT increased human hippocampal volume and ECT-induced volume increases were age-dependent, being more prominent in younger individuals [11]. Since the hippocampus is not a uniform structure and consists of several subfields with distinct morphology, including the cornu ammonis (CA), DG, and subiculum [12], and given the previous evidence of subfield-specific changes, investigating the effect of ECT on hippocampal subfields is warranted. To the best of our knowledge, there are only a few human studies investigating the effect of ECT on hippocampal subfields [13, 14]. Abbott et al. reported in $15 \mathrm{MDD}$ patients that responders to mean 11 ECT sessions showed increased volumes in certain hippocampal subfields (CA1, CA2/3, subiculum, and DG) in the right hemisphere. Cao et al. also reported in 12 MDD patients on remission after 8 ECT sessions showed increased volume in the bilateral granule cell layer $(G C L)$ in the $D G$ and several hippocampal subfields (CA3, CA4, molecular layer, and subiculum) in the right hemisphere. While hippocampal volume increases in the DG after ECT is supported by these prior studies, the clinical relevance of this volume change is still unclear. In addition, the long-term effects of ECT on DG volume are elusive.

To expand on prior findings related to ECT-induced hippocampal volume changes, we conducted the present study. The aims of the current study were to investigate: (1) whether ECT may increase the volume in the DG, (2) whether ECT-induced volume changes in the DG are correlated with demographic variables (e.g. age) and/or clinical remission, and (3) whether volume changes in the DG are transient or permanent. Based on consistent findings from animal work, we hypothesized that ECT would increase DG volume and that clinical improvement might be associated with

\footnotetext{
${ }^{1}$ Department of Neuropsychiatry, Keio University School of Medicine, Tokyo 160-8582, Japan; ${ }^{2}$ Center for Psychiatry and Behavioral Science, Komagino Hospital, Tokyo $193-8505$, Japan; ${ }^{3}$ Cerebral Imaging Center, Douglas Mental Health University Institute, Quebec, Montreal H4H 1R3, Canada; ${ }^{4}$ Department of Biological and Biomedical Engineering, McGill University, Quebec, Montreal H3A 0G4, Canada; ${ }^{5}$ Institute of Medical Science, Faculty of Medicine, University of Toronto, Toronto M5S 1 A8, Canada; ${ }^{6}$ Multimodal Imaging Group -

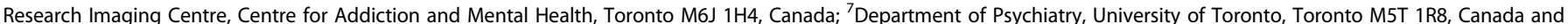
${ }^{8}$ Geriatric Mental Health Division, Centre for Addiction and Mental Health, Toronto M6J 1H4, Canada Correspondence: Taishiro Kishimoto (tkishimoto@keio.jp)
}

Received: 24 July 2018 Revised: 27 December 2018 Accepted: 30 December 2018 Published online: 8 January 2019 
1806

this volume change. According to our meta-analysis [11], we hypothesized that ECT-induced volume increases are negatively associated with age. In addition, we hypothesized that structural changes induced by ECT might be associated with long-term clinical improvement.

\section{PATIENTS AND METHODS}

Participants and clinical assessments

Participants were inpatients scheduled to receive clinically indicated ECT in the Komagino Hospital (a 500-bed psychiatric hospital) in Tokyo, Japan. Healthy controls were recruited from the same area. Written informed consent was obtained from all participants and/or their surrogate family members. This study was approved by the ethics committee of the hospital and performed in accordance with the Declaration of Helsinki. Inclusion criteria were as follows: (1) Diagnostic and Statistical Manual of Mental Disorders, Fourth Edition, Text Revision (DSMIV-TR) diagnosis of major depressive disorder (MDD), or bipolar (BP) I or II disorder experiencing a major depressive episode (MDE) with melancholic features confirmed by Mini-International Neuropsychiatric Interview (M.I.N.I.); (2) clinical indications for ECT, including pharmacotherapy resistant or requiring a rapid response; (3) age $\geq 50$ years. The age range was selected based on a previous similar study [13]. We included only late-life melancholic patients, who have been considered as good candidates for ECT, to minimize heterogeneity in our sample. Exclusion criteria included: (1) Any concurrent Axis I diagnosis other than MDD or BP (e.g. anxiety disorders); (2) concurrent drug or alcohol dependence; (3) a diagnosis of neurological or degenerative disorders (e.g. epilepsy and dementia); (4) unstable or severe medical illness (e.g. myocardial infarction within 1 month); (5) pregnancy; (6) ECT treatment within the last 3 months; (7) patients with mandatory admission by mayor of municipality.

Clinical and MRI assessments were conducted within 1 week before the first ECT (TP1), within 2 weeks (mean 7.3 days) after the last ECT (TP2), and 3 months after the last ECT (TP3). For post-ECT assessments, we avoided at least the first 5 days after the last ECT to minimize any immediate effect of the seizure on imaging and cognitive assessments [13, 15]. Depressive symptoms were evaluated using the 17-item Hamilton Depression Rating Scale (HAM-D). Remission was defined as HAM-D total score $\leq 7$. The Mini Mental State Examination (MMSE) was used to assess global cognitive function. Medication changes and additional ECT treatments based on clinical decisions were permitted during the time between TP2 and TP3. Healthy controls were screened by using the M.I.N.I. and were excluded if they had a history of psychiatric disorders. Healthy controls received 2 assessments at similar intervals (TP1 and TP2) to patients.

\section{ECT procedure}

ECT treatment was provided in the clinical settings by each participant's attending psychiatrist. The participation in our study did not influence any clinical decision for ECT. ECT was performed using a brief-pulse $(0.5 \mathrm{~ms})$ square-wave device (Thymatron system IV device; Somatics, Inc., Lake Bluff, IL, USA). Participants received bilateral ECT 2 to 3 times a week. The number of sessions was determined by each attending psychiatrist. The initial stimulation intensity was determined by half age method (e.g. A 60 -year-old patient received the first ECT at $30 \%$ stimulus output of the machine). Electroencephalogram (EEG) was recorded to ensure at least 20 -sec of epileptiform EEG activity after ECT. Propofol (1 mg/kg) was used for general anesthesia, and succinylcholine $(0.5-1.0 \mathrm{mg} / \mathrm{kg})$ was used to induce muscle relaxation. Participants continued their psychotropic medications throughout the course of ECT. Detailed ECT procedure is described in supplementary material.
Image acquisition and hippocampal segmentation Imaging (MRI) data were acquired using a 3-Tesla GE Signa HDxt scanner at Komagino Hospital. High-resolution 3D T1-weighted images were acquired using a fast spoiled gradient recalled echo sequence (fSPGR: TR $=6.9 \mathrm{~ms}, \mathrm{TE}=2.9 \mathrm{~ms}$, sagittal orientation, matrix $=256 \times 256 \mathrm{~mm}$, slice thickness $=1.0 \mathrm{~mm}$, voxel size $=$ $0.9 \times 0.9 \times 1.0 \mathrm{~mm}^{3}, 174$ slices).

We used a stand-alone analysis pipeline, namely a multiatlas segmentation tool (MAGeT-Brain), to obtain volumes in the CA4/ DG. MAGeT-Brain was demonstrated to show similar results to manual segmentation in standard $\mathrm{T} 1$-weighted images, and to reliably reproduce segmentation for the CA4/DG subfields [16]. In addition, MAGeT-Brain is reported to be more accurate and likely to underestimate the change if any; thus, we considered it is more suited to examine the volume-change longitudinally. A comprehensive description of the segmentation pipeline was provided in the previous articles $[17,18]$. Subfield volumes were normalized for differences in total brain volume (TBV) (gray matter volume plus white matter volume) among subjects by means of an analysis of covariant approach [19]: volume (adjusted) = volume (observed) - Beta * (TBV - mean TBV). Beta was calculated from a linear regression using observed brain volume in the entire sample as the dependent variable and TBV as the independent variable. TBV was obtained using statistical parametric mapping (SPM) 12 package (http://www.fil.ionucl.ac.uk/). We examined the test-retest reliability of automated hippocampal subfield segmentation (supplementary material; Table S1).

\section{Statistical analysis}

To explore cross-sectional effects of diagnosis at baseline (ECT patients and controls), we used a general linear model (GLM), controlling for age and sex.

To confirm ECT-induced CA4/DG volume increases between TP1 and TP2, and to explore the potential effects of clinical characteristics (age and sex) on volume change, we used repeated-measure analysis of variance (RM-ANOVA). To investigate the relationship between clinical improvement and volume changes with ECT, we compared percentage volume changes between TP1 and TP2 among 3 groups (remitters, nonremitters, and controls) using a GLM, including age and sex as covariates, and we performed pair-wise post-hoc comparisons between groups. As additional analyses, we explored the effects of the number of ECT and the dosage of antidepressant medications on volume changes in the CA4/DG (supplementary material). We also explored the correlation between percentage volume changes and percentage change in the clinical ratings (HAM-D and MMSE) (supplementary material).

To investigate whether ECT-related structural changes are transient or not, we used general linear mixed models (GLMM), including time points (TP1, TP2, and TP3) for longitudinal analysis using patients' data only. Age and sex were included in this model as covariates. We selected the GLMM because this model is robust to randomly missing data points and the inclusion of all available data with missing data points is permitted. Dependent variables included percentage volume change in the right and left CA4/DG as primary analyses, and we investigated whether group (i.e. remitters, nonremitters) by time (i.e. TP1, TP2, and TP3) interactions were significant. We reanalyzed data from participants who achieved remission through the entire follow-up period (complete remitters). We also conducted the same analyses including medication dosage (i.e. antidepressants, antipsychotics, benzodiazepine, and mood stabilizer), and lithium therapy (yes/no) as covariates.

Statistical analyses were performed using SPSS ver 24.0 (IBM Inc., Armonk, NY, USA) and R ver 3.4.3. Statistical significance was defined by a $p$-value of $<0.05$ (two-tailed). Bonferroni correction was used for multiple comparisons. For our primary outcomes (volume change in the right and left CA4/DG), statistical 
Table 1. Clinical characteristics of participants

\begin{tabular}{|c|c|c|c|c|c|}
\hline & Controls & \multicolumn{4}{|l|}{ ECT patients } \\
\hline Number & 20 & 25 & 18 & 7 & \\
\hline Age (years) & $63.3(7.3)$ & $67.1(8.3)$ & $66.3(9.6)$ & $69.1(3.0)$ & 0.46 \\
\hline Female (\%) & $13(65.0)$ & $18(72.0)$ & $14(77.8)$ & $4(57.1)$ & 0.36 \\
\hline Bipolar disorder (\%) & & $5(20.0)$ & $3(16.7)$ & $2(28.6)$ & 0.60 \\
\hline Duration of the current episode (month) & & $6.0(3.0-11.0)$ & $8.0(3.0-14.5)$ & $4.0(2.0-5.0)$ & 0.09 \\
\hline Illness duration (year) & & $5.0(1.13-19.5)$ & $4.0(1.19-13.5)$ & $28.0(0.75-47.0)$ & 0.25 \\
\hline Number of ECT & & $10.8(1.5)$ & $10.6(1.4)$ & $11.4(1.6)$ & 0.19 \\
\hline Charge $(\mathrm{mC})$ & & $289.1(86.6)$ & $283.1(90.7)$ & $304.7(79.3)$ & 0.59 \\
\hline EEG seizure (seconds) & & $41.3(12.9)$ & $43.6(14.1)$ & $35.3(6.8)$ & 0.15 \\
\hline
\end{tabular}

significance was defined by a $p$-value of $<0.025(0.05 / 2)$. The raw $p$-values were reported. Detailed explanation of the statistical analysis is described in supplementary material.

\section{RESULTS}

Demographics and clinical characteristics

We included 25 ECT patients and 20 healthy controls. All participants completed imaging assessments at TP1 and TP2. Of 25 patients, 23 patients completed assessments at TP3. MRI data from one subject at TP3 were excluded because of a segmentation error. After 1 course of ECT treatment, 18 patients (72.0\%) met the remission criteria. The mean reduction rate of HAM-D was $80.3 \%$. Between TP2 and TP3, 14 remitters at TP2 kept remission, 4 nonremitters at TP2 remitted at TP3, 4 remitters at TP2 relapsed and converted to nonremitters at TP3, and 2 nonremitters at TP2 were not followed at TP3. Demographics of the study participants are summarized in Table 1, and clinical results are summarized in Table 2. There were differences in MMSE score between remitters and nonremitters at TP1 $(t=2.9, \mathrm{df}=20, p=0.009)$, but no differences at TP2 $(t=1.5, \mathrm{df}=23, p=0.14)$. During the time between TP2 and TP3, one patient (nonremitter at TP2) received a course of ECT and then continuation ECT (remitter at TP3). We excluded this patient's data at TP3 because of unusual clinical scenario.

Baseline comparisons between ECT patients and controls There were no significant differences in right and left CA4/DG volumes between ECT patients and healthy controls at baseline (right: $F_{1,41}=0.00, p=0.99$; left: $F_{1,41}=0.03, p=0.87$ ), and between remitters and nonremitters at baseline (right: $F_{1,21}=$ $0.49, p=0.49$; left: $F_{1,21}=0.03, p=0.87$ ) (Table S2).

Volume change between pre-ECT (TP1) and post-ECT (TP2) Bilateral volume in the CA4/DG increased after ECT (right: $F_{1,24}=$ 14.7, $p=0.001$; left: $F_{1,24}=7.8, p=0.01$ ). There was a significant time by age interaction in the right CA4/DG $\left(F_{1,23}=11.0, p=\right.$ $0.003)$, but no significant time by sex interaction $\left(F_{1,23}=0.48, p=\right.$ $0.50)$. Percentage volume change of the right CA4/DG was negatively associated with age $(r=-0.54, \mathrm{df}=23, p=0.006)$
Table 2. Clinical results of the study

\begin{tabular}{llll}
\hline \multicolumn{3}{l}{ ECT patients } & \\
\cline { 2 - 4 } & TP1 & TP2 & TP3 \\
\hline Number & 25 & 25 & 23 \\
HAMD-17 & $33.0(7.1)$ & $6.0(5.2)$ & $7.2(9.3)$ \\
Remission, $n(\%)$ & & $18(72.0)$ & $17(73.9)$ \\
MMSE & $25.8(3.2)$ & $27.6(2.5)$ & $27.8(2.3)$ \\
Antidepressants, $n(\%)$ & $21(84.0)$ & $21(84.0)$ & $19(82.6)$ \\
Antipsychotics, $n(\%)$ & $19(76.0)$ & $19(76.0)$ & $18(78.3)$ \\
Benzodiazepine, $n(\%)$ & $4(16.0)$ & $4(16.0)$ & $10(43.5)$ \\
Mood stabilizer, $n(\%)$ & $1(4.0)$ & $1(4.0)$ & $5(21.7)$ \\
\hline HAMD-17 and MMSE are described as mean (SD) & \\
\hline
\end{tabular}

(Fig. 1). There were neither significant time-by-age $\left(F_{1,23}=1.56\right.$, $p=0.22)$ nor time-by-sex $\left(F_{1,23}=1.50, p=0.23\right)$ interactions in the left CA4/DG.

In GLMs, a group (remitters, nonremitters, and controls) difference in percentage volume increase was found in the right CA4/DG $\left(F_{2,40}=11.0, p<0.001\right)$ (Fig. 2). Post-hoc comparisons revealed that remitters showed greater volume increases in the right CA4/DG than nonremitters $\left(F_{1,21}=10.28, p=0.004\right)$ and controls $\left(F_{1,34}=21.30, p<0.001\right)$. In contrast, no group differences were found in the left CA4/DG $\left(F_{2,40}=3.15, p=0.05\right)$. The results did not change after controlling the dosage of antidepressant medications and the number of ECT (Figure S4; Figure S5). There were no linear correlation between percentage volume change in the CA4/DG and percentage change in the clinical ratings, including HAM-D and MMSE (supplementary material).

We also performed the same analyses for other hippocampal subfields and whole hippocampus as exploratory analyses. There were significant time by age interactions in the right $\left(F_{1,23}=6.88\right.$, $p=0.015)$ and left $\left(F_{1,23}=7.26, p=0.013\right)$ whole hippocampus (Table S3). Significant group differences in percentage volume increase were found in the right hippocampus $\left(F_{2,40}=5.74, p=\right.$ $0.006)$, the left hippocampus $\left(F_{2,40}=3.89, p=0.03\right)$, and the left 


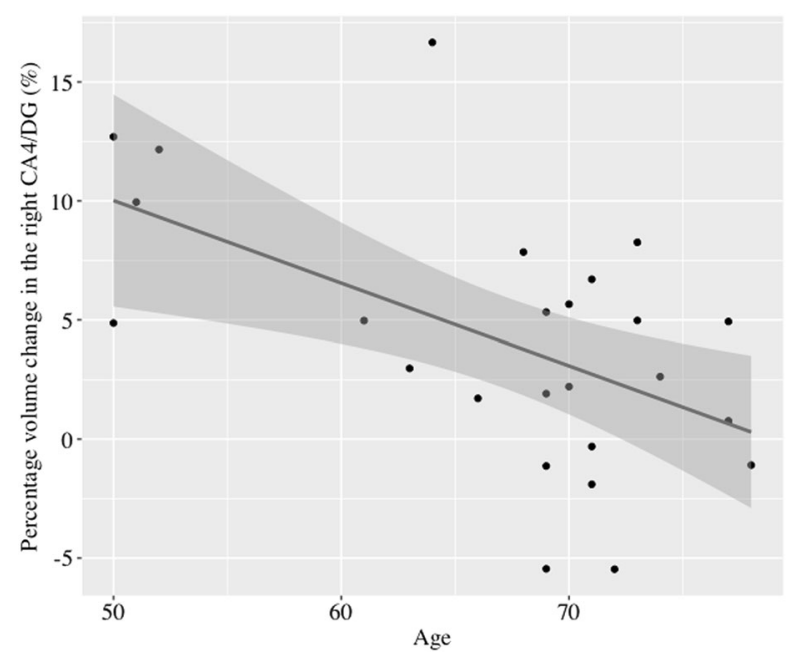

Fig. 1 The relationship between percentage volume changes in the right CA4/DG with ECT and age. Age was negatively associated with volume changes in the right CA4/DG $(r=-0.54$, $\mathrm{df}=23, p=0.006)$. The blue line represents the linear fit and the shaded region represents $95 \%$ confidence intervals

stratum radiatum $(\mathrm{SR})$ /stratum lacunosum $(\mathrm{SL}) /$ stratum moleculare (SM) $\left(F_{2,40}=4.67, p=0.015\right)$ (Table 3; Figure S1).

Long-term volume changes after ECT

The GLMM for the right CA4/DG showed a group (ECT remitters and ECT nonremitters) by time (TP1, TP2, and TP3) interaction $\left(F_{2,46.0}=5.47, p=0.007\right)$, with volume change through 3 time points in ECT remitters only $\left(F_{2,33.5}=17.1, p<0.001\right)$ (Fig. 3$)$. Posthoc analyses revealed that there were differences in remitters' right CA4/DG volume between TP1 and TP2 $(p<0.001)$, and between TP2 and TP3 $(p<0.001)$, but not between TP1 and TP3 $(p=0.35)$. The GLMM for the right CA4/DG also showed a significant main effect of time $\left(F_{2,44.9}=9.71, p<0.001\right)$. Although we reanalyzed using only data from 14 complete remitters, the results did not change (Figure S2).

The GLMM for the left CA4/DG did not show a group by time interaction $\left(F_{2,45.8}=2.84, p=0.07\right)$ and showed a significant main effect of time $\left(F_{2,46.5}=7.03, p=0.002\right)$. Post-hoc analyses of the left CA4/DG in all ECT patients revealed that there were significant differences between TP1 and TP2 $(p=0.01)$, and between TP2 and TP3 $(p=0.001)$, but not significant differences between TP1 and TP3 $(p=0.29)$. When we reanalyzed using only data from 14 complete remitters, there was no significant main effect of time $\left(F_{2,25.1}=3.36, p=0.05\right)$. All analyses were also performed for other subfields and bilateral whole hippocampi as post-hoc exploratory analyses. The results of the right hippocampus showed a similar pattern to the volume change in the right CA4/DG (Figure S3). The results did not change after controlling for maintenance therapy (supplementary material). The actual volume changes in all hippocampal subfields and whole hippocampus through 3 time points were reported in supplementary material (Table S5).

\section{DISCUSSION}

In the current study, we found the following: (1) ECT increased CA4/DG volume; (2) ECT-induced volume increases in the right CA4/DG were negatively associated with age; (3) Remitted patients after ECT showed larger volume increases in the right CA4/DG than non-remitted patients: (4) ECT-induced volume increases in the right CA4/DG were transient and the volume returned to baseline levels 3 months after the last ECT. To the best of our knowledge, this is the first study that investigates both
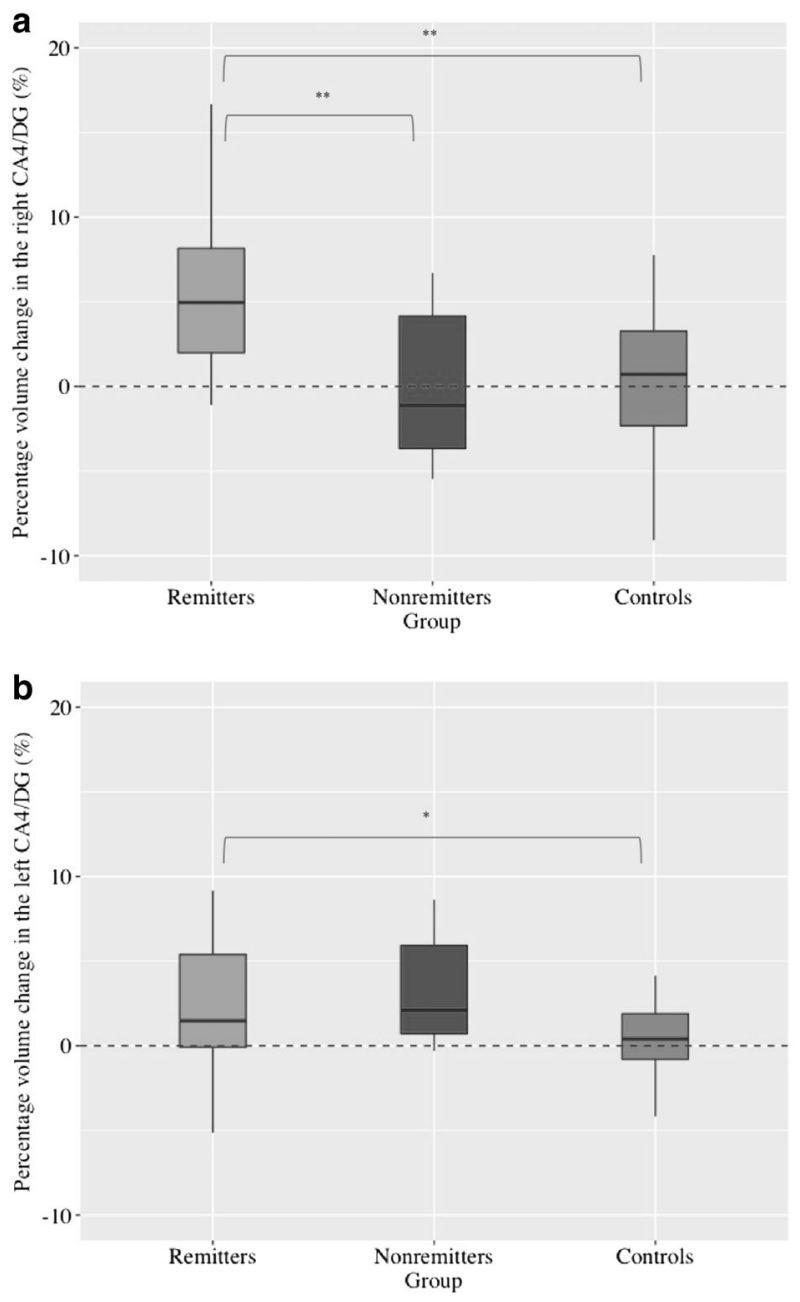

Fig. 2 Longitudinal effect of ECT on the CA4/DG volume. a There were significant group differences in the right CA4/DG volume changes $\left(F_{2,40}=11.0, p<0.001\right)$. Remitters showed significant larger volume increase than both nonremitters $\left(F_{1,21}=10.28, p=0.004\right)$ and controls $\left(F_{1,34}=21.30, p<0.001\right)$. b There were no significant group differences in the left CA4/DG volume changes $\left(F_{2}, 40=3.15\right.$, $p=0.05)$, and post-hoc analyses revealed that remitters showed larger volume increase than controls $\left(F_{1,34}=5.38, p=0.027\right)$. ${ }^{*} p<$ $0.05,{ }^{* *}$ Corrected $p<0.05$

acute ( 1 week) and long-term ( 3 months) effects of ECT on the volume of human hippocampal subfields.

ECT-induced volume increase and age

In this study, we found ECT-induced volume increase in the CA4/ DG. A body of animal studies, including rodent [4] and non-human primate [5] work, has consistently reported that ECS increased neurogenesis in the DG within the hippocampus. Some of the ECS studies reported increased volumes in the DG [7-9]. Similar to ECS, antidepressant medication also induced neurogenesis in the DG [20]. Although both antidepressant medications and ECS induced neurogenesis in the DG, the latter has been shown to be a more potent and faster stimulator of neurogenesis than the former [21]. This evidence seems to be consistent with clinical findings: ECT is a more potent and faster antidepressant treatment than medication. ECS also increases the number of glial cells, endothelial cells, and dendritic spines of neurons [6]. Adult hippocampal neurogenesis in humans has been reported in several studies $[22,23]$, but not all [24]. Given the large volume increase in the CA4/DG (i.e. $\sim 5 \%$ of volume increase), neuroplastic changes other than neurogenesis may mainly account for ECT-induced volume 
Table 3. Results of percentage volume change with ECT among 3 groups (remitters, nonremitters, and healthy controls)

\begin{tabular}{|c|c|c|c|}
\hline & $F$ value & $P$ value & Post-hoc comparisons \\
\hline \multicolumn{4}{|l|}{ Hippocampus } \\
\hline $\begin{array}{l}\text { Right } \\
\text { hippocampus }\end{array}$ & 5.74 & $0.006^{* *}$ & $\begin{array}{l}\text { Remitters }>\text { nonremitters }(p= \\
0.015) \\
\text { Remitters }>\text { controls }(p=0.004)\end{array}$ \\
\hline $\begin{array}{l}\text { Left } \\
\text { hippocampus }\end{array}$ & 3.89 & $0.03^{*}$ & Remitters $>$ controls $(p=0.019)$ \\
\hline \multicolumn{4}{|l|}{ Subfields } \\
\hline Right CA4/DG & 11.0 & $<0.001^{* *}$ & $\begin{array}{l}\text { Remitters }>\text { nonremitters }(p= \\
0.004) \\
\text { Remitters }>\text { controls }(p<0.001)\end{array}$ \\
\hline Left CA4/DG & 3.15 & 0.05 & \\
\hline Right CA1 & 1.39 & 0.26 & \\
\hline Left CA1 & 0.45 & 0.64 & \\
\hline Right subiculum & 0.74 & 0.48 & \\
\hline Left subiculum & 2.09 & 0.14 & \\
\hline Right $C A 2 / 3$ & 1.15 & 0.33 & \\
\hline Left CA2/3 & 1.07 & 0.35 & \\
\hline Right SR/SL/SM & 1.41 & 0.26 & \\
\hline Left SR/SL/SM & 4.67 & $0.015^{*}$ & Remitters $>$ controls $(p=0.005)$ \\
\hline
\end{tabular}

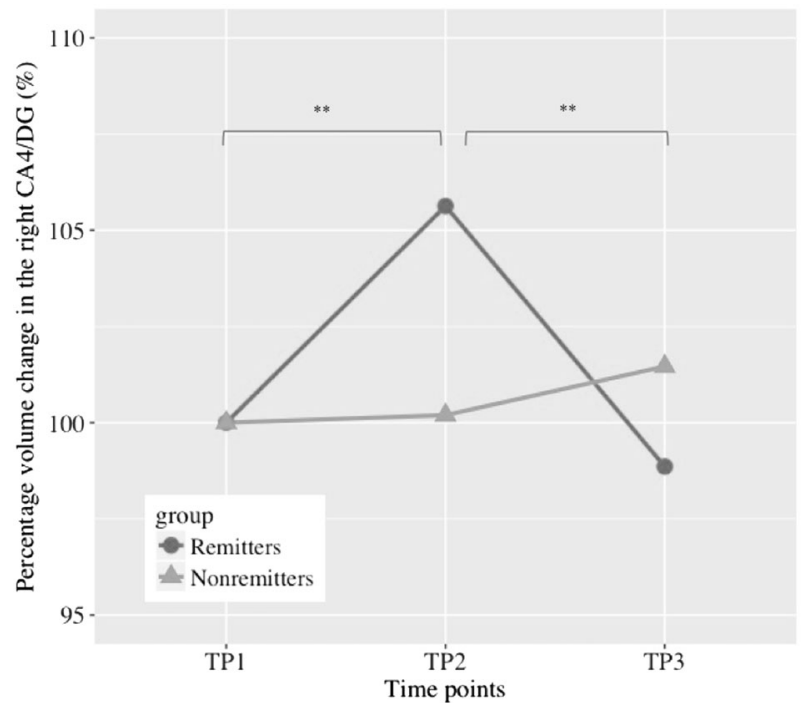

Fig. 3 Long-term volume change in the right CA4/DG. Remitters showed significant volume increase between TP1 and TP2 $(p<$ $0.001)$, but showed significant volume decrease between TP2 and TP3 $(p<0.001)$. There was no significant difference in the right CA4/DG volume between TP1 and TP3 $(p=0.35) .{ }^{*} p<0.05$, ${ }^{* *}$ Corrected $p<0.05$

increases in the CA4/DG detected by MRI. This speculation was supported by a preclinical study, reporting that preclinical deep brain stimulation (DBS)-induced hippocampal volume increase observed on MRI was associated with increased blood vessel size [25].

We found that ECT-related volume increase in the CA4/DG as well as the whole hippocampus was age-dependent, being more prominent in younger patients. The age effect on degree of structural changes was also reported in an ECS study [26]. A recent human study reported elderly individuals had similar numbers of immature neurons in the DG as younger individuals, but had less angiogenesis and neuroplasticity [27]. Hence, age-related CA4/DG volume change with ECT might be due to decline in angiogenesis and neuroplasticity with aging.

Clinical relevance of ECT-related volume change

Larger volume increase in the CA4/DG was found in remitted patients than in non-remitted patients. Preclinical studies showed that the antidepressant effect of ECS was not detected in mice lacking adult neurogenesis [10] and lacking dendritic outgrowth [28]. Antidepressant effect of medications also required neurogenesis [20] or other neuroplastic mechanisms, including dendritic remodeling [29]. Based on these findings, recovery from depression may require neuroplasticity, including neurogenesis, in the DG regardless of treatment modality [30]. The results of the current study could support this speculation, and would highlight novel compounds targeting hippocampal neuroplasticity [31].

According to our systematic review [11], many studies did not find associations between brain structural changes with ECT and clinical improvement. One of the possible explanations for this inconsistency might be due to differences in employed statistical models. We found that the degree of volume change in the CA4/ DG with ECT was negatively associated with age, which was shown to be one of the important predictors for ECT remission [32]. Therefore, we controlled for age when investigating associations between volume change with ECT and clinical improvement. Moreover, we compared remitted patients to nonremitted patients. A recent study also reported only remitted patients showed hippocampal subfield volume increases after ECT [14], although it did not directly compare remitted patients and nonremitted patients. Indeed, similar to previous other studies, we did not find any correlations between percentage change in CA4/ DG volume and percentage change in HAM-D scores. Our results suggest that a categorical approach (i.e. a direct comparison between remitted patients and non-remitted patients) may be meaningful. Although it is speculative, structural plasticity in the CA4/DG may not be the sufficient change, but the necessary change for clinical remission [33]. Another possibility for discrepancies includes a difference in the clinical characteristics of participants. In our cohort, all participants had DSM-defined melancholic features and more than two-thirds had psychotic features. In addition, most of the patients met criteria for melancholia provided by previous research [34] (Table S6). These patients might be representatives of so-called melancholia or an endogenous type of depression [35], which has been considered to reflect more biologically homogenous patients than DSMdefined major depression. These unique characteristics of participants may explain the clear associations between clinical improvement with ECT and hippocampal neuroplasticity, as previously suggested [36, 37].

One might contend that volume changes might be associated with cognitive dysfunction with ECT, not clinical improvement. There are some studies reporting positive associations between hippocampal volume change with ECT and cognitive changes [38] and negative findings $[13,39]$. However, a previous meta-analysis reported that ECT-related cognitive dysfunction was mainly limited to the first 3 days after the last treatment [15], and in this study, we avoided at least the first 5 days after the last ECT for the assessment (all participants received the assessment at least 7 days after the last ECT) to minimize the acute effect on cognition.

Other possibilities include that volume changes might be due to alterations of water content and/or could be just the byproducts of electrical stimulation. However, there are several studies suggesting that edema is unlikely to be the primary cause of the observed hippocampal volume changes with ECT [40-43]. In contrast, there is still a possibility that increased blood flow with 
ECT could affect volume estimation using T1-weighted imaging [44]. Previous studies reported that electrode placement might be related to the side of volume change $[13,45]$, although this is inconsistent $[19,46]$. In the current study, all participants received bilateral stimulation, but volume increase was more evident in the right side than the left side. Our result could raise the possibility that volume change in the CA4/DG was not just the byproduct of stimulation and the right CA4/DG might be more important for remission than the left side. However, we are not able to conclude this yet and future study should include wide varieties of electrode placements in larger cohorts. A recent mega-analysis reported the positive association between the number of ECT and volume increases in the hippocampus with ECT [45]. In our cohort, we did not find any correlations between the number of ECT and bilateral CA4/DG (Figure S4). This inconsistency might be due to the small range of the number of ECT in our cohort (range: 8-14).

We found differences in MMSE total score at TP1 between remitters and nonremitters. Our result is consistent with a previous antidepressant treatment trial that showed pretreatment neuropsychological differences between remitters and nonremitters in the cohort of late-life depression [47], and nonremitters in our cohort might have abnormal white matter integrity based on a vascular depression hypothesis [47]. Another possibility is that some of the nonremitters might have neurodegenerative processes, which might prevent ECT-induced plastic changes. Late-life depression as a whole did not correlate with amyloid deposition [48], but there is still a possibility that a specific subpopulation (e.g. ECT nonremitters) may have neurodegenerative processes. Future studies, including amyloid and/or tau PET assessment as well as assessment of white matter hyperintensities, are needed to explore the underlying neurobiology of ECT nonremitters.

\section{Long-term effect of ECT on CA4/DG}

Contrary to our hypothesis, we found that ECT-induced volume increase in the CA4/DG was evident after 1 week of the last ECT, but the CA4/DG volume returned to baseline levels after 3 months of the last ECT, even in patients who stayed in remission. These results are not consistent with preclinical studies, which report long-term effects of ECS on neurons [4, 49], but are consistent with a human study that reported normalization of hippocampal volume 6 months after the last ECT [19]. Further, we expanded upon this previous finding in that our results suggest that the hippocampal volume effect of ECT was mostly driven by the CA4/ $D G$, and that the volume changes in the CA4/DG were different between remitters and nonremitters. The current study showed a significant group (remitters and nonremitters) by time (TP1, TP2, and TP3) interaction in the right CA4/DG and the right hippocampus. Our results showed a parallel volume change in the right hippocampus and the right CA4/DG (i.e. volume increased at TP2 and normalized at TP3). In addition, we did not find any significant results in other subfields (Table S4). On the left side, we did not find a significant group by time interaction, but found a significant main effect of time, suggesting that both remitters and nonremitters showed similar volume changes in the left CA4/DG and the left hippocampus. Our finding regarding the ECT's prominent effect on the CA4/DG is supported by previous preclinical studies, which have consistently shown the neuroplastic effects (e.g. neurogenesis) of ECS on the DG [4-10]; our results might support the neuroplastic hypothesis of ECT action [6].

Our findings raised the possibility that ECT-induced CA4/DG volume increase might be related to only transition from the depressed state to the euthymic state, but not related to the longterm antidepressant effect of ECT. Although speculative, ECT may provide a short window of structural remodeling in the acute phase, and volume increase with ECT might reflect this process [50]. Future studies should investigate structural and/or functional changes associated with long-term antidepressant effects, as well as the biological effect of maintenance ECT on brain structure and/or function.

\section{Limitations}

Some limitations should be mentioned, but most of them are due to clinical nature of the study. First, the patients continued their psychopharmacological treatment, and some of the patients changed their medications between TP2 and TP3. Our cohort includes severely depressed patients, which required continuous psychopharmacological treatment to prevent further clinical deterioration. We conducted additional analyses to explore the effects of medications on ECT-related volume changes. Even after including medication dosage as covariates in the statistical models, the results did not change (supplementary material). Second, the small number of non-remitted patients limit the power to detect potential confounding factors between remitted and non-remitted patients.

\section{CONCLUSION}

In conclusion, ECT-induced CA4/DG volume increase in the acute phase was associated with age and clinical remission. These results support the neurotrophic hypothesis of depression [30] and neuroplastic change in the dentate gyrus might mediate some of the short-term beneficial effects of ECT. Future studies should examine the underlying biological mechanisms associated with long-term clinical improvement.

\section{FUNDING AND DISCLOSURE}

This study is supported by Inokashira Hospital Grants for Psychiatriy Research (2016-2017), and Keio University Graduate School Grants for Department of Neuropsychiatry (2017-2018). The authors declare no competing interests.

\section{ACKNOWLEDGEMENTS}

We would like to thank Dr. Tamotsu Watanabe, Dr. Saeko Kaneko, Dr. Takasuke Hayakawa, Dr. Ryosuke Tarumi, Dr. Genki Imagawa, Dr. Takayuki Kakimoto, Dr. Kyosuke Sawada and Dr. Taisuke Yatomi for patients' recruitment. We would like to thank Mr. Shiro Nishikata for acquiring MRI data.

\section{ADDITIONAL INFORMATION}

Supplementary Information accompanies this paper at (https://doi.org/10.1038/ s41386-019-0312-0).

Publisher's note: Springer Nature remains neutral with regard to jurisdictional claims in published maps and institutional affiliations.

\section{REFERENCES}

1. Kellner CH, Greenberg RM, Murrough JW, Bryson EO, Briggs MC, Pasculli RM. ECT in treatment-resistant depression. Am J Psychiatry. 2012;169:1238-44.

2. Dierckx B, Heijnen WT, van den Broek WW, Birkenhager TK. Efficacy of electroconvulsive therapy in bipolar versus unipolar major depression: a meta-analysis. Bipolar Disord. 2012;14:146-50.

3. Kellner $\mathrm{CH}$, Fink $M$, Knapp R, Petrides $G$, Husain M, Rummans $T$, et al. Relief of expressed suicidal intent by ECT: a consortium for research in ECT study. Am J Psychiatry. 2005;162:977-82.

4. Madsen TM, Treschow A, Bengzon J, Bolwig TG, Lindvall $O$, Tingstrom A. Increased neurogenesis in a model of electroconvulsive therapy. Biol Psychiatry. 2000;15:1043-9.

5. Perera TD, Coplan JD, Lisanby SH, Lipira CM, Arif M, Carpio C, et al. Antidepressant-induced neurogenesis in the hippocampus of adult nonhuman primates. J Neurosci. 2007;27:4894-901.

6. Bouckaert F, Sienaert $P$, Obbels J, Dols A, Vandenbulcke M, Stek M, et al. ECT: it's brain enabling effects: a review of electroconvulsive therapy-induced structural brain plasticity. J ECT. 2014;30:143-51. 
7. Wennström M, Hellsten J, Ekstrand J, Lindgren H, Tingström A. Corticosteroneinduced inhibition of gliogenesis in rat hippocampus is counteracted by electroconvulsive seizures. Biol Psychiatry. 2006;59:178-86.

8. Chen F, Madsen TM, Wegener G, Nyengaard JR. Repeated electroconvulsive seizures increase the total number of synapses in adult male rat hippocampus. Eur Neuropsychopharmacol. 2009;19:329-38.

9. Kaae SS, Chen F, Wegener G, Madsen TM, Nyengaard JR. Quantitative hippocampal structural changes following electroconvulsive seizure treatment in a rat model of depression. Synapse. 2012;66:667-76.

10. Schloesser RJ, Orvoen S, Jimenez DV, Hardy NF, Maynard KR, et al. Antidepressant-like effects of electroconvulsive seizures require adult neurogenesis in a neuroendocrine model of depression. Brain Stimul. 2015;8:862-7.

11. Takamiya A, Chung JK, Liang KC, Graff-Guerrero A, Mimura M, Kishimoto T. Effect of electroconvulsive therapy on hippocampal and amygdala volumes: systematic review and meta-analysis. Br J Psychiatry. 2018;212:19-26.

12. Cao B, Passos IC, Mwangi B, Amaral-Silva H, Tannous J, Wu MJ, et al. Hippocampal subfield volumes in mood disorders. Mol Psychiatry. 2017;22:1352-8.

13. Abbott CC, Jones T, Lemke NT, Gallegos P, McClintock SM, Mayer AR, et al. Hippocampal structural and functional changes associated with electroconvulsive therapy response. Transl Psychiatry. 2014;4:e483.

14. Cao B, Luo Q, Fu Y, Du L, Qiu T, Yang X, et al. Predicting individual responses to the electroconvulsive therapy with hippocampal subfield volumes in major depression disorder. Sci Rep. 2018;8:5434.

15. Semkovska M, McLoughlin DM. Objective cognitive performance associated with electroconvulsive therapy for depression: a systematic review and meta-analysis. Biol Psychiatry. 2010;68:568-77.

16. Pipitone J, Park MTM, Winterburn J, Lett TA, Lerch JP, Pruessner JC, et al. Multiatlas segmentation of the whole hippocampus and subfields using multiple automatically generated templates. Neuroimage. 2014;101:494-512.

17. Chakravarty MM, Steadman P, van Eede MC, Calcott RD, Gu V, Shaw P, et al. Performing label-fusion-based segmentation using multiple automatically generated templates. Hum Brain Mapp. 2013;34:2635-54.

18. Plitman E, Patel R, Chung JK, Pipitone J, Chavez S, Reyes-Madrigal F, et al. Glutamatergic metabolites, volume and cortical thickness in antipsychotic-naïve patients with first-episode psychosis: implications for excitotoxicity. Neuropsychopharmacology. 2016;41:2606-13.

19. Bouckaert F, Dols A, Emsell L, De Winter F-L, Vansteelandt K, Claes L, et al. Relationship between hippocampal volume, serum bdnf and depression severity following electroconvulsive therapy in late-life depression. Neuropsychopharmacology. 2016;41:2741-8.

20. Santarelli L, Saxe M, Gross C, Surget A, Battaglia F, Dulawa S, et al. Requirement of hippocampal effects of antidepressants. Science. 2003;805:805-9.

21. Malberg J, Eisch A, Nestler EJ, Suman RS. Chronic antidepressant treatment increases neurogenesis in adult rat hippocampus. J Neurosci. 2000;20:9104-10.

22. Eriksson PS, Perfilieva E, Bjork-Eriksson T, Alborn AM, Nordborg C, Peterson DA et al. Neurogenesis in the adult human hippocampus. Nat Med. 1998;4: 1313-8.

23. Spalding KL, Bergmann O, Alkass K, Bernard S, Salehpour M, Huttner HB, et al. Dynamics of hippocampal neurogenesis in adult humans. Cell. 2013;153:1219-27.

24. Sorrells SF, Pareded MF, Cebrian-Silla A, Sandovai K, Qi D, Kelley KW, et al. Human hippocampal neurogeneis drops sharply in children to undetectable levels in adults. Nature. 2018;555:377-81.

25. Chakravarty MM, Hamani C, Martinez-Canabal A, Ellegood J, Laliberte C, Nobrega JN, et al. Deep brain stimulation of the ventromedial prefrontal cortex causes reorganization of neuronal processes and vasculature. Neuroimage. 2016;125:422-7.

26. Rotheneichner P, Lange S, O'Sullivan A, Marschallinger J, Zaunmair P, Geretsegger $C$ et al. Hippocampal neurogenesis and antidepressive therapy: Shocking relations. Neural Plast. 2014: 723915:14p.

27. Boldrini M, Fulmore CA, Tartt AN, Simeon LR, Pavlova V, Rosoklija GB, et al. Human hippocampal neurogenesis persists throughout aging. Cell Stem Cell. 2018;22:589-99.

28. Chang AD, Vaidya PV, Retzbach EP, Chung SJ, Kim U, Baselice K, et al. Narp mediates antidepressant-like effects of electroconvulsive seizures. Neuropsychopharmacology. 2018;43:1088-98.

29. Bessa JM, Ferreria D, Melo I, Marques F, Cerqueira JJ, Palha JA, et al. The moodimproving actions of antidepressants do not depend on neurogenesis but are associated with neuronal remodeling. Mol Psychiatry. 2009;4:764-73.
30. Samuels BA, Hen R. Neurogenesis and affective disorders. Eur J Neurosci. 2011;33:1152-9.

31. Fava M, Johe K, Ereshefsky L, Gertsik LG, English BA, Biello JA, et al. A phase 1B, randomized, double blind, placebo controlled, multiple-dose escalation study of NSI-189 phosphate, a neurogenic compound, in depressed patients. Mol Psychiatry. 2016;21:1372-80.

32. van Diermen $L$, van den Ameele $S$, Kamperman AM, Sabbe BCG, Vermeulen $T$, Schrijvers $D$, et al. Prediction of electroconvulsive therapy response and remission in major depression: meta-analysis. Br J Psychiatry. 2018;212:71-80.

33. Sartorius A, Demirakca T, Bohringer A, Clemm von Hohenberg C, Aksay SS, Bumb $\mathrm{JM}$ et al. Electroconvulsive therapy induced gray matter increase is not necessarily correlated with clinical data in depressed patients. Brain Stimul. 2018; S1935-861:30418-2.

34. Hyett MP, Breakspear MJ, Friston KJ, Guo CC, Parker GB. Disrupted effective connectivity of cortical systems supporting attention and interoception in melancholia. JAMA Psychiatry. 2015;72:350-8.

35. Parker G, Fink M, Shorter E, Taylor MA, Akiskal H, Berrios G, et al. Issues for DSM-5: whither melancholia? The case for its classification as a distinct mood disorder. Am J Psychiatry. 2015;167:745-7.

36. Bolwig TG and Madsen TM. Electroconvulsive therapy in melancholia: the role of hippocampal neurogenesis. Acta Psychiatr Scand Suppl. 2007;115:130-5.

37. Rasmussen KG. Electroconvulsive therapy and melancholia: review of the literature and suggestions for further study. J ECT. 2011;27:315-22.

38. van Oostrom I, van Eijndhoven P, Butterbrod E, van Beek MH, Janzing J, Donders $\mathrm{R}$, et al. Decreased cognitive functioning after electroconvulsive therapy is related to increased hippocampal volume: exploring the role of brain plasticity. J ECT. 2018;34:117-23.

39. Nordanskog P, Larson MR, Larsson EM, Johanson A. Hippocampal volume in relation to clinical and cognitive outcome after electroconvulsive therapy in depression. Acta Psychiatr Scand. 2014;129:303-11.

40. Nordanskog P, Dahlstrand U, Larsson MR, Larsson E-M, Knutsson L, Johanson A Increase in hippocampal volume after electroconvulsive therapy in patients with depression: a volumetric magnetic resonance imaging study. J ECT. 2010;26:62-67.

41. Jorgensen $A$, Magnusson $P$, Hanson LG, Kirkegaard $T$, Benveniste $H$, Lee $H$, et al. Regional brain volumes, diffusivity, and metabolite changes after electroconvulsive therapy for severe depression. Acta Psychiatr Scand. 2016;133:154-64.

42. Kunigiri G, Jayakumar PN, Janakiramaiah N, Gangadhar BN. MRI T(2) relaxometry of brain regions and cognitive dysfunction following electroconvulsive therapy. Indian J Psychiatry. 2007;49:195-9.

43. Szabo K, Hirsch JG, Krause M, Ende G, Henn FA, Sartorius A, et al. Diffusion weighed $\mathrm{MRI}$ in the early phase after electroconvulsive therapy. Neurol Res. 2007;29:256-9.

44. Franklin TR, Wag Z, Shin J, Jagannathan K, Suh JJ, Detre JA, et al. A VBM study demonstrating 'apparant' effects of a single dose of medication on T1-weighted MRIs. Brain Struct Funct. 2013;218:97-104.

45. Oltedal L, Narr KL, Abbott C, Anand A, Argyelan M, Bartsch H, et al. Volume of the human hippocampus and clinical response following electroconvulsive therapy. Biol Psychiatry. 2018;41:105-14.

46. Joshi SH, Espinoza RT, Pirnia T, Shi J, Wang Y, Ayers B, et al. Structural plasticity of the hippocampus and amygdala induced by electroconvulsive therapy in major depression. Biol Psychiatry. 2016;79:282-92.

47. Sheline YI, Pieper CF, Barch DM, Welsh-Bohmer K, McKinstry RC, MacFall JR, et al. Support for the vascular depression hypothesis in late-life depression: results of a 2-site, prospective, antidepressant treatment trial. Arch Gen Psychiatry. 2010;67:277-85.

48. De Winter FL, Emsell L, Bouckaert F, Claes L, Jain S, Farrar G, et al. No association of lower hippocampal volume with Alzheimer's disease pathology in late-life depression. Am J Psychiatry. 2017;174:237-45.

49. Olesen MV, Wortwein G, Folke J, Pakkenberg B. Electroconvulsive stimulation results in long-term survival of newly generated hippocampal neurons in rats. Hippocampus. 2017;27:52-60.

50. Nordgren M, Karlsson T, Svensson M, Koczy J, Josephson A, Olson L, et al Orchestrated regulation of Nogo receptors, LOTUS, AMPA receptor and BDNF in an ECT model suggests opening and closure of a window of synaptic plasticity. PLoS ONE. 2013;8:e78778. 